\title{
RELACIONES DE GÉNERO EN LA ACTIVIDAD PASTORIL
}

\author{
María Amalia Penabad Camacho \\ Estudiante de la carrera Gestión de Desarrollo sostenible \\ con equidad de género, Universidad Nacional \\ Heredia, Universidad Nacional
}

Recibido 7-XI-2007 • Aceptado 13-XI-2007 • Corregido 19-II-2008

\begin{abstract}
Resumen: La preocupación por la histórica invisibilidad de la mujer en todos los campos social, cultural y económico, motiva la elaboración de este artículo titulado "Relaciones de género en la actividad pastoril”. Se expone un marco de referencia general basado en la revisión bibliográfica en la que se describen las relaciones de género en la actividad pastoril.

Se muestran los resultados y el análisis de la información que surge de la revisión bibliográfica y que indica aún una marcada invisibilidad de la mujeres en diversos campos; pero también se encontró que existen varias iniciativas para cambiar este rumbo. Además, se considera que un diagnóstico participativo con enfoque de género, va a plantear diversas acciones estratégicas y concretas para enfrentar la exclusión y discriminación de las mujeres en el pastoreo. Se citan algunos ejemplos sobre esto como el que existe por parte de la FAO, con el Proyecto Forestal IDA-FAO Holanda titulado "Diagnóstico Rural Rápido Participativo con enfoque de Género: Guía Metodológica”.
\end{abstract}

Palabras clave: Pastoreo, relaciones de género, impacto ambiental sostenible.

\section{Revisión bibliográfica sobre las relaciones de género en la actividad pastoril}

La preocupación por la histórica invisibilidad de la mujer en todos los campos social, cultural y económico, motiva una revisión bibliográfica para conocer sobre las relaciones de género en la actividad pastoril.

La división genérica del trabajo en la finca, el hogar y la comunidad, da cuenta de la multiplicidad de funciones que cumplen las mujeres en las actividades agropecuarias y de la invisibilidad que históricamente las ha afectado.

Interesa conocer el estado de la cuestión de la relaciones de género en la actividad pastoril con el objeto de que su aporte sea reconocido y lograr la superación de la subordinación, para que ella trabaje en igualdad de condiciones donde ocupe papeles de liderazgo, reciba capacitación, tome decisiones (Mantilla y otros, 1994, p . 6).

Es claro que el trabajo doméstico es concebido como "de exclusiva competencia de las mujeres", de ahí el recargo en horas de trabajo, cuando además del trabajo reproductor se suma el trabajo productivo. 


\begin{abstract}
The concerns for the historical woman invisibility in social, cultural and economic spaces motivates the origin of this article named: The relation ships of gender in the pastoral activity.

This article is based on a bibliographical compilation. It also shows the results and the analysis of the information that comes from that bibliographical compilation.

This information reveals the profound level of women's invisibilization in the different spaces but also describes a few actions to change it.

A participative with a gender focused diagnosis provides new strategies to fight against women's exclusion and discrimination.

Some examples on this topic are cited from the FAO, the Forestal Project IDA-FAO and Holland named: "Participative Fast Rural Diagnosis with gender emphasis: Methodological Guide.Some excerpts from the article "Diagnóstico Rural Rápido Participativo con enfoque de Género: Guía Methodological" are also considered here.
\end{abstract}

Key words: Shepherding, gender relationships, environmental sustainable impact.
A diferencia de las mujeres, los hombres cumplen únicamente la jornada de trabajo productivo. El reconocimiento por parte de los hombres y las mujeres de la jornada reproductiva, lleva a darle valor a este trabajo y a que se inicie la superación de la subordinación por parte de las mujeres (Ibid., p. 7).

\section{La actividad pastoril y la participación femenina}

La actividad pastoril es un oficio tan antiguo como la existencia del individuo mismo.

Hace 5.000 años, en la época neolítica, comenzó a practicarse el pastoreo. Es, por lo tanto, un oficio muy antiguo en la tierra y así lo prueban muchas cosas; entre otras, el que los actuales pastizales se encuentren en lugares donde hay monumentos megalíticos, o el de que al rico se le llame aberatsa -el que tiene muchos animales- (Los pastores se enfadan, aparecen los quesos, s.f. parr. 1).

El pastoreo es desde el punto de vista económico, el modo de producción basado en las pasturas blandas y la tierra fértil que, permite pastar al ganado. El pastoreo surgió luego de que el hombre cazador, desarrollara técnicas de acorralamiento y así garantizar la carne segura sin los avatares e incertidumbres de la caza.

Con el pastoreo surgieron los primeros conocimientos de agricultura pastoril y el sedentarismo de la especie humana en localidades fértiles.

Junto al pastoreo, que se da en el paso del paleolítico al neolítico, también aparece el filo cortante para cuerear y usar pieles y otras partes del animal, como también, y esto es sumamente importante, aparece el animismo como primer esbozo de creencias en fuerzas supra humanas o religiosas y la reducción del arte imitativo y naturalista a simples formas geométricas (Qué es el pastoreo (s.f.), parr. 1, 2, y 3).

\section{¿Qué es el pastoreo?}

El término Pastoreo es el acto de "llevar los ganados al campo y cuidar de ellos mientras pacen". (Real Academia Española. 2001, p. 1967). El acto de pastorear implica el uso de grandes hectáreas de terreno con pasto para ser desarrollado; que incluye entre ellos: los pastos, el bos- 
que abierto, los matorrales y los desiertos. Debido a que el pastoreo es una práctica muy aplicada en el ámbito agropecuario, esta puede generar impactos ambientales, algunos de los cuales pueden ser negativos para el ambiente.

\section{¿Qué es el impacto ambiental?}

El impacto ambiental se entiende como:

El efecto que produce una determinada acción humana sobre el medio ambiente en sus distintos aspectos... Las acciones humanas, motivadas por la consecución de diversos fines, provocan efectos colaterales sobre el medio natural o social. Mientras los efectos perseguidos suelen ser positivos, al menos para quienes promueven la actuación, los efectos secundarios pueden ser positivos y, más a menudo, negativos..." (Wikipedia. La enciclopedia libre, s.f., parr. 1 y 2).

\section{Relaciones de género en la actividad pastoril}

En la práctica del pastoreo se invisibiliza la participación de las mujeres y por lo tanto no existe una equidad de género en los hábitos pastoriles:

A las mujeres se les consideraba meras beneficiarias del desarrollo en función del papel reproductivo que jugaban en la economía mientras que sus roles productivos como el de la agricultura, no se tomaban en cuenta (Braidotti, 2004, p. 25).

Es por lo tanto necesario visibilizar los impactos negativos que produce el pastoreo en el ambiente y destacar el aporte femenino en este tipo de actividades para generar de esta forma una equidad de género.

Hay que visibilizar las contribuciones de las mujeres en este tipo de procesos, ya que parte de la discriminación generada por su género se debe a que se tienen concepciones de que la mujer no da un gran aporte a la sociedad cuando es ella quien dedica mayor inversión de tiempo en contribuir con el desarrollo.
Además de esto, es necesario conocer la relación que une al género con el ambiente para sensibilizarse sobre los daños causados a éste y así generar cambios que contribuyan a un mejor desarrollo humano sustentable.

Es por estas razones que el trabajo se propone identificar las características de las relaciones de género en la actividad pastoril sostenible por medio de una revisión bibliográfica; conceptualizar una práctica pastoril sostenible con dimensión de género y reflexionar sobre el impacto ambiental generado por el pastoreo y su impacto diferenciado por género, para dar respuesta al problema ¿Cuáles son las relaciones de género en la actividad pastoril?

\section{Género y antecedentes}

Género es una construcción social en la que se dan diferentes características definidas a partir del sexo de la persona ya sea mujer u hombre.

La humanidad debe preocuparse por resolver el problema de discriminación de las mujeres. Es necesario generar conciencia acerca de las formas en que se ha creado discriminación hacia las mujeres para que así se puedan eliminar estos prejuicios que son formados con el aprendizaje social. El solo hecho de que existan inequidades en la vida social, imposibilita el derecho de todas las personas a ser iguales en todo aspecto. Es por esto necesario: establecer condiciones de igualdad de trato, desarrollar políticas de igualdad de oportunidades e impulsar una educación igualitaria".

Desde el punto de vista de la ecología feminista se tiene que:

La ecología política feminista considera el género como una variable crítica que conforma el acceso a los recursos, y su control, al interactuar con la clase, la casta, la raza, la cultura, y la etnicidad para, así, dar forma a procesos de cambio ecológico, a la lucha de los hombres y las mujeres para sostener formas de subsistencia ecológicamente viables y a las expectati- 
vas que cualquier comunidad tiene de un "desarrollo sustentable” (Rocheleau y otras, 2004, p. 345).

\section{El género y el ambiente: buscando una sostenibilidad}

Para dar a conocer el posible origen de las relaciones entre el género y el ambiente, hay que explicar el surgimiento de algunas organizaciones que buscaban la sustentabilidad del ambiente para generar desarrollo. Mujeres en Desarrollo (MED O WED) fue una estrategia puesta en práctica por organismos internacionales que buscaron "incorporar al desarrollo" a mujeres de países subdesarrollados o del tercer mundo (conocidos como países del Sur).

El tema WED por sus siglas en inglés (Mujeres en Desarrollo) se originó en el contexto del desarrollo económico de los países del Sur, esto es, en las discusiones sobre las mujeres en el desarrollo y el medio ambiente y el desarrollo (Braidotti, 2004, p. 23).

Como resultado de los cuestionamientos fundamentales del ambientalismo el tema WED se amplió y las mujeres y los hombres que trabajaban en el campo de la asistencia al desarrollo comenzaron a preguntarse sobre la sustentabilidad del desarrollo en el Sur (Braidotti, 2004, p. 24).

Sin embargo, a pesar de entender el papel que ocupaba la mujer en la producción, sólo se tomaba en cuenta su papel reproductivo.

Durante las décadas de 1950 y 1960, las organizaciones que se ocupaban del desarrollo únicamente consideraron el papel que las mujeres tenían en la reproducción .... (Braidotti, 2004, p. 24).

A las mujeres se les consideraba meras beneficiarias del desarrollo en función del papel reproductivo que jugaban en la economía mientras que sus roles productivos como el de la agricultura, no se tomaban en cuenta (Braidotti, 2004, p. 25).

Según López (2005, parr. 2), comprenderemos mejor si pensamos que en aquellas primeras décadas para el desarrollo, a partir de los sesenta, las mujeres eran percibidas únicamente como receptoras de bienes y servicios y se consideraba que su único papel posible en el desarrollo era la reproducción social: ocuparse de las tareas domésticas, del cuidado de los hijos y mayores y de la atención a sus familias... Puesto que ellas quedaban fuera de la esfera de lo "públicoproductivo", había que "integrarlas" en el desarrollo, había que promover una "mayor participación de las mujeres en el desarrollo económico”.

Ester Boserup publica en 1970, su libro "El papel de la mujer en el desarrollo económico" [Woman' role in Economic Development] citado por Braidotti y explica que:

Ellas no sólo se benefician automáticamente de los programas de desarrollo, sino que, por el contrario, el proceso de desarrollo a menudo ha tenido como consecuencia el deterioro relativo y hasta absoluto de la posición de las mujeres frente a los hombres; son ellos quienes de manera creciente son incluidos en los sectores agrícolas que se van modernizando, en tanto que ellas continúan formando parte de la agricultura de subsistencia y no tienen acceso a créditos, capacitación, ni tecnología y concluye que la introducción de nuevos métodos agrícolas tuvo efectos negativos en las mujeres del Sur porque cambió los patrones de la división sexual del trabajo y las desplazó de sus áreas tradicionales de trabajo (Braidotti, 2004, p. 25).

López (2005 parr. 3) cita a Boserup e indica que esta escribe un libro producto de una amplia investigación, desconocida hasta el momento, de la contribución de las mujeres al área productiva de la economía. $\mathrm{Y}$ de esto, emergieron dos consecuencias para el desarrollo: la justificación para que los proyectos de desarrollo también fueran dirigidos a las mujeres como productoras y la crítica a la forma en que se habían llevado a cabo hasta entonces tales proyectos, pues habían comportado un deterioro en la condición y posición de las mujeres.

Surgen organizaciones como GAD (siglas en inglés para referirse a género y desarrollo) en la cual:

...se busca la igualdad total de las mujeres en el marco del desarrollo económico y GAD es importante debido a que ...es un elemento importante para conseguir la igualdad de género.... (Braidotti, 2004, p. 30).

Según Braidotti (2004), debido a diferentes problemas que surgieron como crisis 
de petróleo, escasez de recursos naturales y la gran deforestación que "se debía sobre todo a la tala de los bosques que tenía propósitos comerciales y a la extensión de la agricultura a dichas tierras..." (p. 33), la UNEP (siglas en inglés para referirse al Programa de las Naciones Unidas para el medio ambiente) y la WED (siglas en inglés para referirse a mujeres, medio ambiente y desarrollo), comenzaron programas para el mejor manejo ambiental con participación de las mujeres y así se desarrollaron varias estrategias de desarrollo con enfoque de género.

\section{Iniciativas como estrategia para resolver el problema de la invisibilidad de las mujeres}

Ya existe el esfuerzo por parte de la FAO, con el Proyecto Forestal IDAFAO Holanda titulado "Diagnóstico Rural Rápido Participativo con enfoque de Género: Guía Metodológica”.

Mantilla, Madden y León. Diagnóstico Rural Rápido Participativo con enfoque de género: Guía Metodológica (1999), p. 7, presentan el "Resultado de la sistematización del proceso de Diagnóstico Rural Rápido Participativo con enfoque de género (DRRPG), que el proyecto IDA-FAO realizó en la Región Chorotega, Provincia de Guanacaste en Costa Rica, durante los años 96-97, con el acompañamiento de la Asociación andar, una ONG costarricense, que trabaja por el desarrollo rural desde 1989" y señalan que:

El proceso de diagnóstico participativo con enfoque de género, que realizó el proyecto IDA-FAO con la participación de las mujeres y los hombres de las distintas comunidades que participaron, demuestra un avance no sólo en los aspectos metodológicos y de técnicas, sino también de concepción, pues el género no se había visualizado anteriormente como una variable a ser tomada en cuenta en los Diagnósticos Rurales Rápidos Participativos (Ibíd.).

Además los autores verifican los avances del DRRPG, puesto que
Toma en cuenta los principios y la fundamentación teórica que se sustenta en la participación de las comunidades, como gestoras de su propio desarrollo, clarificando en su planteamiento que las relaciones sociales entre las mujeres y los hombres es clave para el desarrollo de ambos géneros en particular y para la sociedad en general. En el planteamiento se reconoce la discriminación que históricamente han sufrido las mujeres, no sólo por su condición de género, sino también por otros factores como la edad, la etnia o la clase social (Ibid., p. 8).

\section{¿Qué es el DRRPG?}

\section{El DRRPG consiste en}

Un estudio de la realidad económica, social y cultural de una comunidad, orientado a identificar sus principales problemas y necesidades, descubrir las causas de los mismos y definir líneas de acción que orienten la búsqueda de soluciones. Puede ser utilizado para investigar diferentes aspectos relacionados con el desarrollo de las comunidades: salud, vivienda, extensión agrícola, agroforestería, mejoramiento del medio ambiente, etc. (Ibíd., p. 9).

\section{El DRRPG con enfoque de género}

Se utiliza para contribuir al desarrollo de las comunidades propiciando la igualdad de oportunidades para ambos géneros (Ibid., p. 11).

El DRRPG con enfoque de género se fundamenta en los principios de sostenibilidad ecológica, viabilidad económica y consenso social y favorece los procesos sociales que se caracterizan por la equidad, la igualdad y la autonomía entre los géneros (Ibid., p. 11).

Por otra parte, no sólo se debe resolver la inclusión de género, pues también sobre el tema tenemos otra disyuntiva referida a los efectos negativos que genera el pastoreo, sobre el que se ampliará a continuación.

\section{Efectos negativos del pastoreo}

La deforestación para generar ganado se ha vuelto una práctica muy grande en América Central y América del Sur. El manejo de las tierras deforestadas no es 
adecuada, lo que provoca el agotamiento de la fertilidad del suelo, daño de su estructura y se disminuye el terreno pastoril, lo que a su vez genera un ciclo en el que los productores tienen que talar más árboles y se provoca un daño en el ecosistema.

Parte de los principales daños derivados del pastoreo lo constituye el consumo excesivo, con la degradación de la vegetación y erosión de los suelos.

\section{Pastoreo sostenible}

Debido a la gran deforestación que se produce a causa del pastoreo, la FAO (siglas en inglés para referirse a la Organización de las Naciones Unidas para la Agricultura y la Alimentación), está implementando en países como Nicaragua, Colombia y Costa Rica la práctica de pagar a los criadores de ganado para que planten árboles, arbustos forrajeros y setos vivos en los pastizales donde pasta el ganado. Existe un proyecto llamado "pago por los servicios ambientales", en el que participan hasta el momento 450 agricultores y se les premia por el mejor uso de la tierra. Este proyecto da inicio en el 2002, con apoyo del Banco Mundial y la Iniciativa para Ganadería, Medio Ambiente y Desarrollo (LEAD) y su objetivo es el de:

“...estudiar estrategias de producción pecuaria en zonas de pastoreo degradadas, y experimentar si el pago de servicios ambientales puede desincentivar la difundida deforestación que se lleva a cabo para abrir pastizales" (FAO, 2006, p. 1).

Por medio de sistemas silvostorales que consiste en la plantación de arbustos forrajeros árboles y pastos mejorados; se está tratando de restituir el nitrógeno al suelo, además de proveer más nutrientes, lo que genera un hábitat sostenible y reduce la erosión y el desprendimiento de sedimentos. Según el coordinador de LEAD, Hennig Steinfield:

Los ganaderos y los agricultores pueden beneficiarse considerablemente... Los sistemas silvopastorales una vez establecidos pueden sustentar a muchos más animales por hectárea que los pastizales naturales o mejorados. El forraje y la fruta de los árboles y los arbustos también proporcionan al ganado una alimentación más nutritiva, con lo cual aumenta la producción de leche y carne (FAO, 2006, p. 1).

Pero el problema de este sistema está en la desinformación de la gente y la falta de dinero para invertir, ya que no se tiene idea del valor ambiental de esta práctica y no se cuenta con los recursos económicos ni el tiempo para invertir en ella.

Para que los productores pecuarios adopten esta medida, se les incentiva con esta propuesta de "pago por los servicios ambientales" y resulta una actividad muy rentable, ya que dependiendo de las toneladas de carbono que sean fijadas, así serán remunerados. Todo esto hace que se valore más la tierra y se busquen medidas para enriquecerla. El objetivo es que la práctica del silvopastoreo sea más rentable que las prácticas de pastoreo tradicionales.

La práctica del silvopastoreo ha traído muchos beneficios para Costa Rica, ya que además de aportar con cuantiosas sumas de dinero a los productores que aplican la siembra de árboles en áreas de pastoreo, esta ha contribuido a generar proyectos para la mejora del ambiente.

Este trabajo se propone hacer una búsqueda bibliográfica para identificar las relaciones de género en la actividad pastoril.

El objetivo consiste en identificar las características de las relaciones de género en esta actividad para lo cual se pretende hacer:

- una revisión bibliográfica,

- $\quad$ conceptualizar una práctica pastoril sostenible con dimensión de género y - reflexionar sobre el impacto ambiental generado por el pastoreo.

Mediante un enfoque cualitativo se estudian los significados de las acciones humanas y de la vida social, en este caso, 
se analizan las relaciones de género en las actividades pastoriles. Es de tipo bibliográfico, descriptivo, ya que su objetivo es la descripción de fenómenos y usa los estudios correlacionales y de desarrollo, además, se basa en la información que existe sobre los temas del pastoreo y de género.

\section{Conclusiones}

La revisión bibliográfica realizada permite concluir los efectos negativos que provoca el pastoreo mal aplicado en el ambiente y su impacto.

Impacto ambiental se conceptualiza como:

“conjunto de posibles efectos negativos sobre el medio ambiente de una modificación del entorno natural, como consecuencia de obras u otras actividades" (Real Academia Española. 2001, p. 1251).

En el caso del pastoreo, el impacto ambiental es provocado por la actividad de la deforestación, la cual se ha convertido en una práctica muy frecuente en América Central y América del Sur.

El manejo de las tierras deforestadas no es adecuada, con lo cual se provoca el agotamiento de la fertilidad del suelo, daño de su estructura y se disminuye el terreno pastoril, lo que a su vez genera un ciclo en el que los productores tienen que talar más árboles y con el consecuente daño en el ecosistema.

Parte de los principales perjuicios producto del pastoreo es el consumo excesivo, lo que conduce a una degradación de la vegetación, erosión de los suelos.

Teóricamente tenemos que desarrollo sostenible se refiere a:

... la capacidad de la humanidad para garantizar que satisface las necesidades actuales sin arriesgar la capacidad de que las generaciones futuras satisfagan sus propias necesidades (Cuervo, Luis E. (s.f.).

En la revisión bibliográfica encontramos, en referencia al tema que nos ocupa, que existen varias iniciativas como estrategia para resolver el impacto ambiental provocado por el pastoreo en el desarrollo sostenible, según la FAO.

El proyecto "pago por los servicios ambientales", mencionado en páginas anteriores para llevar la práctica del pastoreo sostenible es una adecuada solución a la degradación que se está provocando en el ambiente producto de la deforestación descontrolada. Sin embargo, esta propuesta no aplica la inclusión de género. Se hace necesario que se planteen para este proyecto iniciativas en las que se valore el aporte femenino, ya que de esta forma se estaría dando un mayor alcance al desarrollo humano sostenible.

De acuerdo con la revisión bibliográfica se puede determinar que hay escasa inclusión de género en las actividades de pastoreo.

A las mujeres se les consideraba meras beneficiarias del desarrollo en función del papel reproductivo que jugaban en la economía mientras que sus roles productivos como el de la agricultura, no se tomaban en cuenta" (Braidotti, 2004, p. 25).

Dentro de los textos consultados sobre el pastoreo, no se refleja un lenguaje inclusivo, además de que en los planes de acción planteados no se toma en cuenta la participación femenina. Sin embargo, ya se pueden citar algunos esfuerzos sobre dicho tema.

A pesar de que la mujer sí ha participado de las labores de pastoreo, este trabajo no es visibilizado. Dentro esta dinámica el papel de la mujer no es ajeno a la actividad productiva, pues aunque es invisibilizado ella está contribuyendo de manera activa.

Ya existe el esfuerzo por parte de la FAO, con el Proyecto Forestal IDA-FAO Holanda titulado "Diagnóstico Rural Rápido Participativo con enfoque de Género: Guía Metodológica”. En dicho Proyecto Mantilla, Madden y León (1999, p. 7), presentan el "Resultado de la sistematización del proceso de Diagnóstico Rural Rápido Participativo con enfoque de género (DRRPG) y señalan 
que una de las principales características consiste en que es:

Un proceso sistemático y coordinado que requiere de la participación activa de los hombres y mujeres de la comunidad, que son quienes deciden los temas básicos a investigar, la forma de investigarlos y el uso de los resultados de la investigación (Ibid., p. 19).

El diagnóstico participativo con enfoque de género toma en cuenta los problemas de los hombres y las mujeres de la comunidad e identifica las diferencias entre ambos géneros en cuanto a intereses y necesidades. Analiza la posición de la mujer en relación con el hombre y determina si ésta es dependiente o subordinada (Ibid., p. 20).

Se concluye que aún existe una marcada invisibilización de la mujer en los campos socio-económicos y culturales, puede determinarse que no hay inclusión de género en las actividades de pastoreo. Y no se refleja un lenguaje inclusivo. En los planes de acción no hay participación femenina. Sin embargo, ya hace algunos años se notan esfuerzos por solucionar este panorama y un ejemplo de ello es el esfuerzo por parte de la FAO, con el Proyecto Forestal IDA-FAO Holanda titulado "Diagnóstico Rural Rápido Participativo con enfoque de Género: Guía Metodológica”, sistematizado por los especialistas Mantilla, Madden y León, en el que se considera que partir de un diagnóstico participativo con enfoque de género, va a plantear diversas acciones estratégicas y concretas para enfrentar la exclusión y discriminación de las mujeres en el pastoreo.

\section{Recomendaciones}

La propuesta de Pastoreo Sostenible, Visibilizar el aporte de la mujer a las actividades de pastoreo.

Promover la inclusión de la perspectiva de género en los hábitos pastoriles actuales.

Determinar posibles soluciones que contrarresten el efecto negativo del pastoreo en el ambiente.
Generar campañas de información sobre los hábitos sostenibles de pastoreo con enfoque de género.

Aplicar a los planteamientos existentes sobre el pastoreo sostenible un enfoque de género.

\section{Referencias bibliográficas}

Barrantes, R. (2006). Paradigmas de la Investigación Científica. En R. Barrantes (Aut.), Investigación: un camino al conocimiento, un enfoque cualitativo y cuantitativo (pp. 53-73). San José, EUNED.

Braidotti, R. (2004). Mujeres, medio ambiente y el desarrollo sustentable: surgimiento del tema y diversas aproximaciones. En L. Madden Arias (Comp.), Antología del curso Medio ambiente y equidad de género (pp. 27-49). San José, UNA.

Campillo, F. (1994). Género y desarrollo rural sostenible: hacia una relación de mayor igualdad. En L. Madden Arias (Comp.), Antología del curso Medio ambiente y equidad de género (pp. 27-49). San José, UNA.

Cuervo, L. E. (s.f.). Nuestro futuro común. Extraído el 12 de Octubre, 2007 de http://www.flora.org/sustain/ Espanol-/WCED.shtml

Lamas, M. (1996, Enero-Marzo). La perspectiva de género [Versión electrónica]. La tarea. Revista Educación y Cultura, 8, sp.

López M., I. (2005). Género negado, vivido y la política de desarrollo. El concepto de género y sus implicaciones para el desarrollo. Extraído el 20 de noviembre del 2007 de http://www.ehu.es/pazydesarrollo/working/working08.htm 
Los pastores se enfadan, aparecen los quesos [Artzaiak samur, gasnak ager]. (s.f.). Extraído el 11, Octubre, 2007 de http://www.geocities.com/Athens/ Bridge/2252/pastoreo.htm

Mantilla, J., Madden, L. y León, R. (1999). Diagnóstico Rural Rápido Participativo con enfoque de Género, Guía Metodológica. [DRRPG]. San José, Costa Rica: Proyecto Forestal IDA-FAO-HOLANDA.

Organización de las Naciones Unidas para la Agricultura y la Alimentación [FAO]. (2006, Marzo). Pastoreo sostenible. Italia: Departamento de Agricultura, Bioseguridad, Nutrición y Protección del Consumidor. Extraído el 11 de Octubre, 2007 de http://www.fao.org/ag/esp

Qué es el pastoreo. (s.f.). Extraído el 11 de Octubre, 2007 de http://espanol.answers.yahoo.com/question/ index?qid=20071117085359AAoID7a
Real Academia Española. (2001). Diccionario de la Lengua Española (22 ${ }^{\circ}$ ed., Tomo II). España: Espasa Calpe.

Rocheleau, D., Thomas-Slayter, B., y Wangari, E. (2004). Género y ambiente: una perspectiva de la ecología política feminista. En: V. Vázquez García y M. Velásquez Gutiérrez (Coords.), Miradas al futuro: hacia la construcción de sociedades sustentables con equidad de género ( $p$. 345). México, D. F.: Universidad Nacional Autónoma de México, Centro Regional de Investigaciones Multidisciplinarias. Programa universitario de Estudios de Género.

Wikipedia, La enciclopedia libre. (s.f.). Impacto ambiental. Extraído a las 23:45, del 9 de febrero, 2008 de http://es.wikipedia. org/w/index.php?title=Impacto ambiental\&oldid=14988009 
